increases of $36 \%, 25 \%$ and $42 \%$ respectively. Among the women, at age $38,16.8 \%$ reported ever having a SSP, and $22.7 \%$ ever a SSE. Based on responses from all assessments, the respective proportions were $20.5 \%$ and $31.4 \%$, increases of $22 \%$ and $45 \%$ respectively.

Conclusions In this cohort SSB was more common that reported from other population-based studies. There are three plausible explanations for the marked discrepancies between the lifetime SSB reported at age 38 and derived from all reports: (1) some reports at early assessments were erroneous, (2) with age participants were less willing to report past same-sex behaviour, (3) with age earlier SSB were forgotten or reinterpreted as not being sexual. The latter seems most plausible.

Disclosure of interest statement None of the authors have conflicts of interests to disclose.

\section{P11.02 REPRESENTING MEN WHO HAVE SEX WITH MEN (MSM) IN BRITAIN: EVIDENCE FROM COMPARATIVE ANALYSES OF THE LATEST CONVENIENCE AND PROBABILITY SURVEYS}

${ }^{1} \mathrm{P}$ Prah*, ${ }^{2} \mathrm{~F}$ Hickson, ${ }^{1} \mathrm{C}$ Bonell, ${ }^{3} \mathrm{~L}$ McDaid, ${ }^{1,2} \mathrm{~B}$ Erens, ${ }^{3}$ J Riddell, ${ }^{1} \mathrm{~S}$ Wayal, ${ }^{4} \mathrm{~A}$ Nardone, ${ }^{1} \mathrm{P}$ Sonnenberg, ${ }^{1} \mathrm{AM}$ Johnson, ${ }^{1} \mathrm{CH}$ Mercer. ${ }^{1}$ University College London; ${ }^{2}$ London School of Hygiene and Tropical Medicine; ${ }^{3}$ University of Glasgow; ${ }^{4}$ Public Health UK

\subsection{6/sextrans-2015-052270.452}

Introduction Convenience samples of MSM are typically skewed towards gay-identified and community-attached men who do not necessarily represent all MSM, a key population for STI/HIV transmission. Comparing convenience samples with a probability sample of MSM enables us to assess their representativeness.

Methods We compared 148 MSM in Britain's third National Survey of Sexual Attitudes and Lifestyles (Natsal-3), with men in Britain's key convenience surveys of gay men: 15,500 in the European MSM Internet Survey (EMIS), 1,012 in the Gay Men's Sexual Health Survey London (GMSHS-London), 1,234 in the Gay Men's Sexual Health Survey Scotland (GMSHS-Scotland), all undertaken 2010-2012. Men were aged 18-64, resident in Britain, and reported $>=1$ male sexual partners (past year). Comparisons include demographic, health characteristics and sexual behaviours, using identically-worded questions. Multivariable analyses accounted for socio-demographic differences between the samples.

Results Convenience samples had significantly younger and better educated men than Natsal-3, and a larger proportion of men identifying as gay (88\%-95\% vs. 62\%). Partner numbers were larger and same-sex anal sex more commonly reported by convenience samples but no significant differences were observed in age at first sex or reporting unprotected anal intercourse. Compared to Natsal-3, men in convenience samples were more likely to report HIV testing (past year) (all adjusted odds ratios (AORs) $>=2.30,95 \%$ CI lower bounds $>=1.49$ ) and gonorrhoea diagnoses (all AORs $>=7.99$, 95\% CI lower bounds $>=1.11$ ). However, fewer differences between samples were observed when analyses focused on MSM who identified as gay.

Conclusion Participation bias may mean convenience samples of MSM demographically misrepresent and over-estimate sexual activity and HIV testing for the entire MSM population. However, they may be more representative of gay-identified MSM, capture a broader range of behaviours and achieve larger samples, relative to general population surveys. Methods are needed to triangulate data from different surveys to strengthen the evidence-base for MSM.
P11.03 LUBRICANT USE DURING RECEPTIVE ANAL INTERCOURSE AND RECTAL CHLAMYDIAL AND GONOCOCCAL INFECTIONS AMONG MEN WHO HAVE SEX WITH MEN

${ }^{1} \mathrm{C}$ Maierhofer* ${ }^{*}{ }^{1} \mathrm{CE}$ Rice, ${ }^{2} \mathrm{KS}$ Fields, ${ }^{2} \mathrm{M}$ Ervin, ${ }^{3} \mathrm{AN}$ Turner. ${ }^{1}$ Division of Epidemiology, College of Public Health, The Ohio State University, Columbus, OH, USA; ${ }^{2}$ Sexual Health Clinic, Columbus Public Health, Columbus, OH, USA; ${ }^{3}$ Division of Infectious Diseases, Department of Internal Medicine, College of Medicine, The Ohio State University, Columbus, $\mathrm{OH}$, USA

\subsection{6/sextrans-2015-052270.453}

Introduction Despite the frequent use of lubricants during anal sex, few studies have examined associations between specific lubricant types and rectal STIs.

Methods Between July 2012 and October 2013, we conducted a cross-sectional study of men who have sex with men (MSM) recruited from an urban STD clinic in the Midwestern United States. In a self-administered survey, participants identified the types and frequency of lubricants used during receptive anal intercourse (RAI) in the previous three months. Rectal chlamydial and gonococcal infection was diagnosed using nucleic acid amplification testing. Among men who report any RAI in the previous three months, we used multivariable logistic regression models to analyse associations between recent use of nine specific lubricants and prevalent rectal infection (chlamydia or gonorrhoea).

Results Of 235 MSM in the study, $77 \%(n=182)$ reported RAI in the previous three months, and $95 \%$ of those $(\mathrm{n}=173)$ reported lubricant use during RAI during the same time period. In separate, unadjusted models, Gun Oil (OR: 2.11, 95\% CI: 1.04, 4.25), Slick (OR: 4.35, 95\% CI: 1.27, 14.89), baby oil (OR: 4.35, 95\% CI: 1.27, 14.89), Vaseline (OR: 2.53, 95\% CI: 1.08, 5.91), and precum (OR: 2.74, 95\% CI: $1.25,6.01)$ were each associated with prevalent rectal chlamydia or gonorrhoea. After adjustment for age, condom use, number of partners, and HIV status, only precum remained significantly associated with prevalent rectal infection (AOR: 3.10, 95\% CI: 1.15, 8.38).

Conclusion Rectal lubricant is a very common exposure among MSM, a population with high prevalence of STIs and HIV. In unadjusted analyses, we observed significant associations between several lubricants and rectal infection, however after multivariable adjustment, only precum was associated with prevalent infection. Longitudinal studies are needed to examine any causal relationship between specific lubricants and STI acquisition.

Disclosure of interest statement No authors have any conflict to disclose.

\section{P11.05 PROPORTION OF HIV AND SEXUALLY TRANSMITTED INFECTION (STI) CASES AMONG MEN WHO HAVE SEX WITH MEN (MSM) ATTRIBUTABLE TO SEROSORTING}

${ }^{1} \mathrm{CM}$ Khosropour* ${ }^{*},{ }^{1,2}$ LA Barbee, ${ }^{1,2} \mathrm{JC}$ Dombrowski, ${ }^{1,2}$ RP Kerani, ${ }^{1,2}$ DA Katz, ${ }^{3} \mathrm{~F}$ Swanson, $1,2 \mathrm{MR}$ Golden. 'University of Washington; ${ }^{2}$ Public Health - Seattle \& King County; ${ }^{3}$ Gay City Health Project

\subsection{6/sextrans-2015-052270.454}

Introduction The contribution of serosorting to HIV/STI disease burden among MSM is unknown.

Methods The study used data collected as part of routine care at an STD clinic 2002-2013 or a community-based HIV/STD testing centre 2004-2013 in Seattle, Washington. Data included men's condom use for anal sex with HIV-positive, negative and 
unknown-status partners in the prior 12 months. We defined three mutually-exclusive behavioural categories: serosorting (condomless anal intercourse [CAI] only with HIV-concordant partners); non-concordant CAI (any CAI with HIV-discordant/ unknown-status partners; [NCCAI]); and no CAI (consistent condom use or no AI). We estimated adjusted relative risks (aRR) between sexual behaviour and HIV/STI, and calculated the population attributable risk (PAR\%) as the proportion of HIV/STI cases attributable to serosorting (assuming serosorters would have otherwise consistently used condoms) and the population prevented fraction (PF) as the proportion of hypothetical HIV/STI cases averted by serosorting (assuming serosorters would have otherwise had NCCAI).

Results Behavioural data were complete for 49,912 visits; 91\% $(\mathrm{n}=45,220)$ were among self-reported HIV-negative MSM. Overall, serosorting was reported by $35 \%$ and $38 \%$ of HIV-negative and positive MSM, respectively. Compared to men reporting no CAI, HIV-negative serosorters had a significantly higher risk of HIV $(\mathrm{aRR}=2.0)$, syphilis $(\mathrm{aRR}=2.0)$, urethral gonorrhoea/chlamydia $(\mathrm{GC} / \mathrm{CT})(\mathrm{aRR}=1.5)$ and rectal GC/CT $(\mathrm{aRR}$ $=1.7$ ). The proportion of HIV/STI cases attributable to serosorting (PAR\%) were: HIV (15.3\%); syphilis (16.9\%); urethral GC/CT (11.9\%); rectal GC/CT (19.3\%). Serosorters had a significantly lower risk of $\mathrm{HIV}(\mathrm{aRR}=0.53)$ and syphilis $(\mathrm{aRR}=$ 0.76) compared to men reporting NCCAI; the proportion of HIV and syphilis cases averted by serosorting (PF) were $14.6 \%$ and $8.3 \%$, respectively. Among HIV-positive MSM, syphilis risk was significantly higher for serosorters compared to consistent condom users $(\mathrm{aRR}=1.4$; PAR\% $=12.1 \%)$.

Conclusion These data suggest that serosorting is responsible for, but also prevents, $15 \%$ of HIV cases among MSM (PAR $\%$ $=15.3 \%$ and $\mathrm{PF}=14.6 \%$ ), depending on the behaviour it replaces.

Disclosure of interest statement No pharmaceutical grants were received in the development of this study.

\section{P11.07 THE SPECTRUM OF SEXUAL BEHAVIOURS AMONG MSM AND ASSOCIATIONS WITH PREVALENT SEXUALLY TRANSMITTED INFECTIONS AND HIV}

${ }^{1} \mathrm{CE}$ Rice, ${ }^{1} \mathrm{AH}$ Norris, ${ }^{2} \mathrm{JA}$ Davis, ${ }^{3} \mathrm{CD}$ Lynch, ${ }^{4} \mathrm{KS}$ Fields, ${ }^{4} \mathrm{M}$ Envin, ${ }^{2} \mathrm{AN}$ Turner${ }^{*} .{ }^{1}$ Division of Epidemiology, College of Public Health, The Ohio State University, Columbus, OH, USA; ${ }^{2}$ Division of Infectious Diseases, Department of Internal Medicine, College of Medicine, The Ohio State University, Columbus, OH, USA; ${ }^{3}$ Department of Obstetrics and Gynecology, College of Medicine, The Ohio State University, Columbus, OH, USA; ${ }^{4}$ Sexual Health Clinic, Columbus Public Health, Columbus, OH, USA

\subsection{6/sextrans-2015-052270.455}

Introduction "Sex" among men who have sex with men (MSM) encompasses a much broader range of behaviours than just anal intercourse, yet most sexual health research in this population focuses on only this one practice. We undertook this study to more fully characterise the spectrum of sexual behaviours endorsed by MSM seeking care in an urban, public STD clinic and the associations between these behaviours and prevalent STI and HIV.

Methods This cross-sectional study of 235 MSM was conducted from July 2012 through October 2013. Participants self-administered a standardised survey assessing several categories of sexual behaviour, including multiple types of receptive/insertive behaviours, venues where sexual activity occurs, and sex while using drugs and alcohol. Men responded about behaviours over their lifetime and within the past three months. We generated unadjusted prevalence ratios (PRs) to characterise associations between recent participation in each behaviour and prevalent STI and HIV.

Results Participants' median age was 26 years. Most were white $(57 \%)$ and employed (73\%). Most, but not all, identified as gay $(76 \%)$. One-third $(35 \%)$ had rectal or urethral gonorrhoea or chlamydia, or primary/secondary syphilis. STI prevalence was significantly higher among men who reported using sex slings (PR: 2.04), anonymous sex (PR: 1.81), illicit drugs during sex (PR: 1.77), fisting (PR: 1.74), sex toys (PR: 1.44), and group sex (PR: 1.43). HIV prevalence was $17 \%$, and was significantly higher among men who endorsed fisting (PR: 4.81), felching (PR: 3.92), enemas (PR: 4.04), sex slings (PR: 2.47), drug use during sex (PR: 1.98), and group sex (PR: 1.92).

Conclusions Many behaviours other than anal intercourse were significantly associated with prevalent STI and HIV in unadjusted analyses, demonstrating the need for prospective studies to examine causal relationships between specific behaviours and STI or HIV acquisition.

Disclosure of interest statement No authors have any conflict to disclose.

\section{P11.08 KNOWLEDGE OF SEXUALLY TRANSMITTED DISEASE RISK AMONG MEN WHO HAVE SEX WITH MEN}

Salil C*, E Jeptanui. Department of Sexology, Information Centre for HIV Prevention, Moi Teaching and Referral Hospital, Eldoret, Kenya

\subsection{6/sextrans-2015-052270.456}

Introduction Sexually Transmitted Diseases (STDs) are the most frequent among Infectious diseases. In pathogenesis of the diseases that can be transmitted during sexual intercourse among, the most important is the problem of infected Anal secretions' transfer. That transfer may happen directly during the Anal's mucosa. The objective of the studies was risk awareness on STDs knowledge among men who have sex with men (MSM).

Methods To describe the problem, the studies were done on 50 men of which $60 \%$ were MSMs, and $30 \%$ were Bisexual. The survey was carried out using internet, through the web page for Homosexuals. The research was based on Personal Questionnaire; concerning demographical data, question about sexuality and knowledge of STD.

Results Almost half of the Men participating in the studies considered the level of their knowledge of STDs infection prevention were good. Most of them (ca.75\%) reported that has never suffered an STD before. About 52\% of the participants had problems to define infection risk in different sexual practices correctly, as well as to point out infection tracts. The biggest problem for them was to connect typical symptoms with particular diseases. However almost all participants assumed any STD's symptoms appearance is an indication for immediate medical attention.

Conclusions Although the prevailing parts of the participants were convinced to be well -informed about STDs, their knowledge was not deep - what can increase STDs infection risk. 